\title{
Preface
}

\section{Cardiac Arrhythmias in Adults with Congenital Heart Disease}

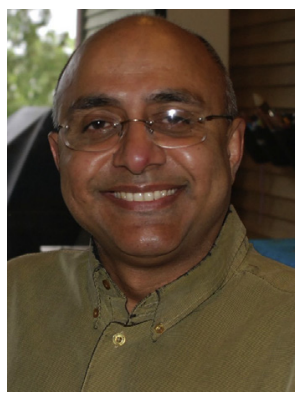

Seshadri Balaji, MBBS, FRCP(UK), PhD

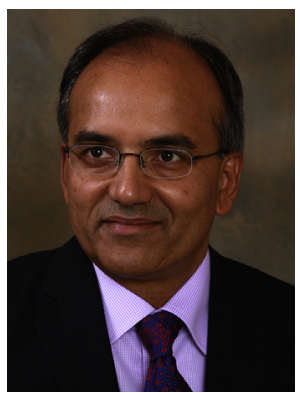

Ravi Mandapati, MD, FHRS, FACC

Editors
This issue of Cardiac Electrophysiology Clinics is entirely devoted to Arrhythmias in Adults with Congenital Heart Disease. It is intended to be a comprehensive and up-to-date review of the state of this rapidly growing field. The material is aimed toward adult and pediatric cardiologists who come across older children and adults with congenital heart disease.

As more and more children survive heart surgery and their life expectancy improves, there are now more adults than children alive with congenital heart disease, and most of them have repaired congenital heart disease. Rhythm disorders are among the most prominent complications encountered by adults with congenital heart disease. Arrhythmias are the leading cause of mortality and morbidity in this population.

The range of complexity of congenital heart disease and the innovative surgeries that have been performed create challenges for the future management of these patients. For one, it is hard to see homogenous cohorts with similar disease that can be systematically studied. While there are a few defects like transposition of the great arteries and tetralogy of Fallot that tend to be relatively homogenous, others, particularly more complex defects like single ventricle, tend to be

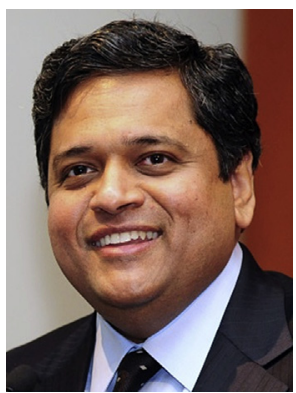

Kalyanam Shivkumar, MD, PhD, FHRS

highly variable in the range and complexity of the anomaly from one patient to another. The lack of a homogeneous group that can be studied leads to the problem of most rare diseases: namely, that clinical research tends to be based on small case collections rather than large-scale studies. Thus, the management of these patients tends to vary from center to center based on local experience and expertise. Also, many of the management protocols are based on similar diseases and conditions studied in larger populations. Electrophysiologists who care for such patients develop opinions and dogmas based on our understanding of the underlying pathophysiology of arrhythmias in such complex patients. Given this problem, it is not hard to see why there are multiple approaches to the same condition and some repetition and even disagreement among experts.

Advances in catheter ablation technologies, image integration, and so forth, have revolutionized management for many arrhythmias. An important aspect of the care of adults with congenital heart disease is the nonuniform transition of care from pediatrics to adult cardiology. The growth of adult congenital heart disease as a subspecialty has been patchy and variable from center to center. This has created the tendency for patients to 
"fall off the radar" when they reach college age. Many of them then show up in emergency rooms (ERs) far away from their primary place of care with poor records and a poor self-understanding of their heart condition. When they show up in such an ER with an arrhythmia, the local ER physicians tend to be placed in a highly unfavorable position in caring for these patients. It is beyond the scope of this issue to deal with such transitions of care. It is our hope, however, that this issue becomes a reference manual for practitioners in such a place.

We have been fortunate to have some of the world's experts agree to and expeditiously write articles on their topics of expertise. We, the editors, are extremely indebted to the authors for doing such a terrific job under a stressful deadline.

We dedicate this issue to the countless patients with congenital heart disease, who have taught us valuable lessons and endured our ignorance as we painfully learned about the best ways to care for them over the past few decades.
Seshadri Balaji, MBBS, FRCP(UK), PhD

Pediatric Arrhythmia, Doernbecher

Children's Hospital

Oregon Health and Science University Portland, OR 97239, USA

Ravi Mandapati, MD, FHRS, FACC

Cardiac Electrophysiology

Loma Linda University

International Heart Institute

Loma Linda University Health

11234 Anderson Street

Loma Linda, CA 92354, USA

Kalyanam Shivkumar, MD, PhD, FHRS

UCLA Cardiac Arrhythmia Center

David Geffen School of Medicine

UCLA Health System

Los Angeles, CA 90025, USA

E-mail addresses:

balajis@ohsu.edu (S. Balaji)

rmandapati@llu.edu (R. Mandapati)

KShivkumar@mednet.ucla.edu (K. Shivkumar) 